\title{
A direct stability analysis of a radiation-induced natural convection boundary layer in a shallow wedge
}

\author{
By CHENGWANG LEI AND JOHN C. PATTERSON \\ School of Engineering, James Cook University, Townsville, Queensland 4811, Australia
}

(Received 7 May 2002 and in revised form 29 October 2002)

This study considers the convective instability of a water-filled shallow wedge with an absorptive bottom subject to solar radiation. Previous studies have revealed that a thermal boundary layer develops along the sloping bottom due to the absorption of penetrative radiation there, and this boundary layer is potentially unstable to the Rayleigh-Bénard instability. The stability properties of the thermal boundary layer are determined in the present study by perturbing the three-dimensional numerical solution. The result of the direct stability analysis has confirmed previous scaling with respect to the convective instability. Additional features of the thermal layer instability have also been revealed from the direct stability analysis.

\section{Introduction}

Buoyancy-induced fluid motions in cavities belong to a broad class of flow which forms the basis of a wide range of applications in nature and engineering. Consequently, a large literature exists, especially for the classical cases of rectangular, cylindrical or other regular geometries with an imposed temperature gradient or imposed boundary heat fluxes. Reviews by, for example, Ostrach (1988) and Hyun (1994) give a broad background to these cases.

The particular case of natural convection in triangular cavities has received relatively little attention, and has been motivated by two main applications: the fluid dynamics and heat transfer in an attic space (e.g. Poulikakos \& Bejan 1983a, $b$; Salmun 1995a,b; Asan \& Namli 2000); and the fluid dynamics and mass transfer in nearshore lake waters, reservoir sidearms or other shallow water bodies with a sloping bottom (e.g. Horsch \& Stefan 1988; Horsch, Stefan \& Gavali 1994; Farrow \& Patterson 1993a,b, 1994; Lei \& Patterson 2001, 2002a,b).

In the latter application, a significant mechanism for forcing has been shown to be the daytime unequal capture of radiation as the result of the changing water depth (Monismith, Imberger \& Morison 1990). Put simply, constant surface incident radiation is absorbed by the water column; in the shallow part, the vertically averaged volumetric rate of absorption is greater than that in the deeper part, and a horizontal temperature gradient results with the average temperature increasing with decreasing depth. Consequently, a natural convection flow results, manifested as a warm water surface outflow from the shallow tip, and a deep return flow up the bottom slope.

The actual flow is of course much more complex than this simple description. In particular, the use of the vertically averaged volumetric radiation absorption implicitly assumes that all of the radiation that arrives at the surface is absorbed in the water column, and that the absorption is uniform over the total depth. In reality, neither 
of these assumptions is true. A more realistic model was described by Farrow \& Patterson (1994). Here, the depth-dependent absorption of radiation was taken into account. As well as providing a vertical distribution of absorption and therefore of heating, this also meant that differing intensities of radiation arrived at the bottom surface, depending on depth, and were absorbed by the bottom. This absorbed heat was re-emitted as a boundary heat flux. Since the surface was assumed to be insulated, all of the incoming radiation was ultimately absorbed by the water column, but with a rather different distribution than the simple vertically averaged model.

With this model there is the potential for a thermal instability in the flow, with bottom heating arising from the boundary heat flux. This competes with the stabilizing absorption of radiation through the surface; the depth-dependent absorption means that the upper parts of the water column are heated at a higher rate than the deeper parts, resulting in a stably stratified system.

Lei \& Patterson (2002a) described a series of experiments that demonstrated the natural convection flow resulting from this combination of forcing mechanisms. In those experiments, a cavity with a sloping, absorptive bottom was illuminated on the surface by a theatre spot lamp. The incident radiation penetrated the surface and was partially absorbed by the water column; any radiation remaining at the bottom was re-emitted by the absorptive surface. The flow was visualized with shadowgraph images, and temperatures were measured at a number of discrete locations close to the sloping bottom along its length. The same authors (Lei \& Patterson 2002b) undertook a scaling and a numerical analysis in two dimensions of the same problem and identified a number of flow regimes during the flow development from initiation of the radiation input, consistent with the observations of the experiments. The latter study identified the controlling parameters as the Prandtl number Pr, the Grashof number $G r$ and the cavity aspect ratio $A$ :

$$
\begin{gathered}
\operatorname{Pr}=\frac{v}{k}, \\
G r=\frac{g \beta H_{0} h^{4}}{v^{2} k}, \\
A=\frac{h}{L},
\end{gathered}
$$

where $v$ and $k$ are the kinematic viscosity and thermal diffusivity for water respectively, $g$ is the acceleration due to gravity, $\beta$ is the coefficient of thermal expansion, $h$ and $L$ are the maximum water depth and the length of the domain. $H_{0}$ is the surface heating intensity defined by

$$
H_{0}=\frac{I_{0}}{\rho_{0} C_{p}},
$$

where $I_{0}$ is the surface radiation intensity, $\rho_{0}$ is the water density and $C_{p}$ the specific heat of water. Three main flow regimes were revealed from the scaling analysis for typical bottom slopes: conductive, for $\operatorname{Pr} G r<A^{-2}$ in which a thermal boundary layer along the bottom boundary simply grows to encompass the entire domain; transitional, for $A^{-2}<\operatorname{Pr} G r<A^{4} R a_{c}^{3}$ in which a convective circulation arises as well as the formation of the thermal boundary layer; and convective, for $\operatorname{Pr} G r>A^{4} R a_{c}^{3}$ in which the flow is dominated by convective transport in a strong circulation. Here, the parameter $R a_{c}$ is the critical Rayleigh number for the appearance of Rayleigh-Bénard convection, adjusted for the bottom slope (see Lei \& Patterson 2002b). 
In this last flow regime, a complex set of flow instabilities appeared in both the experiments and numerical simulations (Lei \& Patterson 2001, 2002a), and the scaling analysis was able to give estimates of the time scale for their appearance (Lei \& Patterson 2002b). The primary form of the instabilities observed in the experiments was rising plumes released from the thermal boundary layer (Lei \& Patterson 2002a). Streamwise wave-like structures were also observed in the thermal boundary layer which formed in the deep part and travelled up the slope. The rising plumes that penetrated the stably stratified region in the upper part of the water column contributed significantly to the net heat transfer from the heated bottom to the upper surface.

Both the experiments and numerical simulations clearly demonstrated that the development of the flow in the convective regime passed through three transient stages (Lei \& Patterson 2001, 2002a,b): an initial stage characterized by rapid growth of a thermal boundary layer along the sloping bottom formed by the heat flux from that boundary; a transitional stage characterized by the presence of instabilities forming on the bottom boundary as rising plumes which translated up the slope; and a quasi-steady stage in which there was a steady rise in the average temperature of the fluid, accompanied by a steady circulation out along the surface from the shallow tip and a return flow up the slope, with weak instabilities rising from the bottom boundary but not penetrating to the surface.

The three-dimensional nature of these instabilities was demonstrated by Lei \& Patterson (2001) with a numerical solution of the full three-dimensional governing equations for this case. The three-dimensional solution was also compared with a twodimensional calculation. Interestingly, the two-dimensional simulation reproduced all of the flow features regardless of the actual three-dimensional nature of the flow, indicating that a two-dimensional simulation was, in many respects, a very good model for the actual flow when gross features such as net heat or mass transfer were to be considered. However, the actual details of the instabilities and their interaction could obviously not be recovered from a two-dimensional simulation.

The stability properties of this configuration have been examined in an asymptotic sense by Farrow \& Patterson (1993b). In that paper, the stability properties of an asymptotic solution of the governing equations with $A \rightarrow 0$ were examined, seeking the critical conditions which generated instabilities in the form of rolls with axes aligned in the up-slope direction. The limitations of the asymptotic result, and the observation of three-dimensional structures in the studies referred to above, however, indicate that this approach is not sufficient to characterize the stability properties in a useful way.

In this paper the stability properties of the flow in the initial and transitional stages are examined using the method of direct numerical stability analysis (Armfield \& Janssen 1996; Janssen \& Armfield 1996). Here, the full three-dimensional numerical solution of the developing flow is disturbed by a small-amplitude perturbation, and the propagation of the disturbance is examined. The perturbation may be applied in two ways: first, a perturbation of uniform amplitude and randomly distributed wavenumber; and second, a range of single-wavenumber disturbances of uniform amplitude. Both lead to essentially the same result. In the first case the amplitude spectrum of the resulting signal gives the amplification properties of the flow as a function of wavenumber, and in the second, the amplitudes of the surviving signals at the input wavenumber give the amplification at that particular wavenumber, and a spectrum may be constructed from a range of input wavenumbers. The critical condition, that is where the growth rate is greater than zero, may be determined 


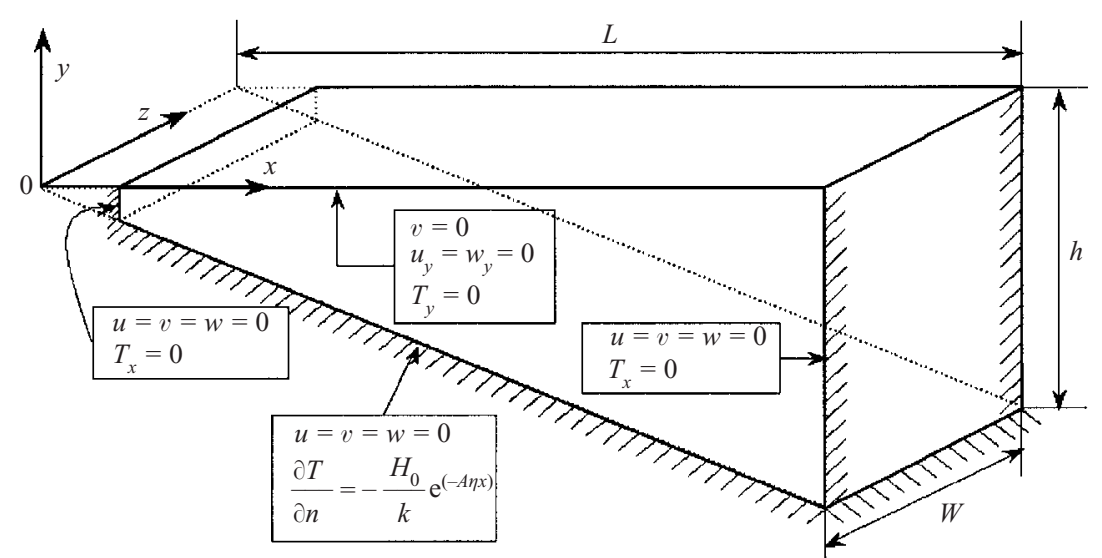

FIGURE 1. A schematic of the geometry with boundary conditions.

by examining the amplitude of the signals, again as a function of wavenumber. The minimum condition for a positive growth rate yields the critical condition.

The remainder of the paper is organized as follows: First, the numerical model and techniques are given in $\S 2$. Preliminary results are then obtained in $\S 3$ to demonstrate the effects of the amplitude of perturbation as well as the grid resolution and time step. Section 4 presents detailed results of the direct stability analysis. In this section, the flow development with the presence of an artificial perturbation is discussed, and the flow responses to both random and single-mode perturbations are investigated. The Grashof number effects are also examined in this section and the critical conditions are identified. Finally, a brief summary is given in $\S 5$.

\section{Formulation and numerical procedures}

\subsection{Model formulation}

A three-dimensional wedge (see figure 1) with rigid non-slip boundaries at the bottom and end and a free surface at the top is considered. The geometry of the wedge is defined by the length $(L)$, the maximum water depth $(h)$ and the width $(W)$ along the transverse direction. The wedge is filled with water initially at rest and at temperature $T_{0}$. At time $t=0$, radiation of intensity $I_{0}$ is initiated and thereafter maintained at the water surface. The attenuation of the radiation travelling through a water column is characterized by Beer's law (Rabl \& Nielsen 1975; Kirk 1986):

$$
I=I_{0} \mathrm{e}^{\eta y} \quad(y \leqslant 0),
$$

where $I$ is the radiation intensity at a given depth, $y$ the negative water depth, and $\eta$ the attenuation coefficient of water. The attenuation coefficient for water is strongly dependent on the wavelength of the radiation and the turbidity of water (Kirk 1986). However, it is assumed here that the absorption of the solar radiation is characterized by a single bulk attenuation coefficient, which is a common assumption in limnological applications. The shallow wedge assumption implies that the water depth is less than the attenuation length of the radiation, i.e. $h<\eta^{-1}$. In this case, a significant amount of radiation reaches the sloping bottom.

The subsequent flow and temperature changes within the wedge are governed by the three-dimensional Navier-Stokes equations and the energy equation with Boussinesq 
assumptions:

$$
\begin{gathered}
u_{t}+u u_{x}+v u_{y}+w u_{z}=-\rho_{0}^{-1} p_{x}+v \nabla^{2} u, \\
v_{t}+u v_{x}+v v_{y}+w v_{z}=-\rho_{0}^{-1} p_{y}+v \nabla^{2} v+g \beta\left(T-T_{0}\right), \\
w_{t}+u w_{x}+v w_{y}+w w_{z}=-\rho_{0}^{-1} p_{z}+v \nabla^{2} w \\
T_{t}+u T_{x}+v T_{y}+w T_{z}=k \nabla^{2} T+H_{0} \eta \mathrm{e}^{\eta y} \\
u_{x}+v_{y}+w_{z}=0,
\end{gathered}
$$

where $x, y$ and $z$ are coordinates along the length, depth and width respectively, $u, v$ and $w$ are the corresponding velocity components, $p$ is the pressure, $T$ is the temperature and $t$ is the time. The subscripts $x, y, z$ and $t$ in the above equations denote partial differentiation with respect to spatial directions and time. The second term on the right-hand side of the energy equation (9) quantifies the direct absorption of radiation by the water body (Farrow \& Patterson 1994).

The temperature and velocity boundary conditions for this problem are described as follows (refer to figure 1 and also Farrow \& Patterson 1994):

(i) Sloping bottom $(y=-A x): u=v=w=0$ (rigid non-slip). Assuming the bottom is absorptive and insulated at the external side results in a boundary heat flux, which is then perturbed. Accordingly, the temperature boundary condition is

$$
\frac{\partial T}{\partial n}=-\frac{H_{0}}{k} \mathrm{e}^{-A \eta x}+P t \quad(y=-A x)
$$

where $n$ is the coordinate normal to the sloping bottom, $P t$ is the artificial perturbation. In this study, two different perturbations are applied:

$$
\begin{aligned}
P t & =\varepsilon[\operatorname{rand}(0,1)-0.5] \frac{H_{0}}{k} \quad \text { (random perturbation) } \\
P t & =0.5 \varepsilon\left[\sin \left(k_{c} z+\phi\right)\right] \frac{H_{0}}{k} \quad \text { (single-mode perturbation), }
\end{aligned}
$$

where $\varepsilon$ specifies the intensity of the perturbation $(\varepsilon \ll 1)$; rand $(0,1)$ generates random numbers between 0 and $1 ; k_{c}$ is the wavenumber in the transverse direction and $\phi$ is the initial phase given by

$$
\phi=2 \pi \operatorname{rand}(0,1) .
$$

(ii) Endwall $(x=L): \partial T / \partial x=0$ (insulated) and $u=v=w=0$ (rigid non-slip).

(iii) Water surface $(y=0): \partial T / \partial y=0$ (no heat loss) and $\partial u / \partial y=\partial w / \partial y=0$, $v=0$ (stress free).

(iv) Sidewalls $(z=0$ and $W): f_{z=0} \equiv f_{z=W}$ (periodic), where $f$ represents any quantity of the flow (temperature, velocity, pressure and their derivatives). The implementation of these conditions in our numerical simulations ensures that up to the second derivatives are equal at the two ends in the transverse direction.

\subsection{Quasi-steady-state simplification and normalization}

Since the water body in the wedge is heated continuously by absorbing the radiation penetrating through the water column, and there is no heat loss through the boundaries, the water temperature will continue to increase without a limit, and there will be no steady state in terms of the temperature. However, with a constant surface radiation applied, a quasi-steady state may be reached in which the temperature increases at the same rate everywhere whereas the temperature gradients and flow 
velocities become steady. The temperature change in the wedge can be split into two components:

$$
T-T_{0}=\bar{T}(t)+\tau(x, y, z, t),
$$

i.e. a spatially averaged temperature, $\bar{T}(t)$, which increases in time, and a spatial variation of temperature, $\tau(x, y, z, t)$, which has a steady-state spatial distribution after a transition. The spatially averaged temperature can be obtained from a balance of the energy entering from the surface and that absorbed by the water body, which gives $\bar{T}(t)=\left(2 H_{0} / h\right) t$, a linear increase with time.

Substituting equation (15) into equations (7) and (9) yields the following equations with respect to $\tau$ :

$$
\begin{gathered}
v_{t}+u v_{x}+v v_{y}+w v_{z}=-\rho_{0}^{-1} p_{y}+v \nabla^{2} v+g \beta(\bar{T}+\tau), \\
\tau_{t}+u \tau_{x}+v \tau_{y}+w \tau_{z}=k \nabla^{2} \tau+\left(H_{0} \eta \mathrm{e}^{\eta y}-2 H_{0} / h\right) .
\end{gathered}
$$

With the Boussinesq assumptions for buoyancy in place, the spatially averaged temperature $\bar{T}(t)$ can be included in the background pressure and therefore neglected in equation (16). Since all boundary conditions for the temperature $(T)$ involve temperature gradients only and are independent of time, the same boundary conditions apply for $\tau$ as for $T$. Equations (9) and (17) are identical except that there is an additional heat sink on the right-hand side of equation (17). This term is used to balance the linear increase of the spatially averaged temperature due to the absorption of radiation. When this balance is achieved everywhere in the domain, a steady state is reached for $\tau$, corresponding to the quasi-steady state for $T$. The complete system of equations now consists of (6), (16), (8), (17) and (10) with the spatially averaged temperature $\bar{T}(t)$ dropped from equation (16).

The quantities in the quasi-steady-system equations are then normalized with the following scales: the length scale $(x, y, z) \sim h$, the time scale $t \sim h^{2} / k$, the temperature variation scale $\tau \sim H_{0} h / k$, the velocity scale $(u, v, w) \sim k / h$, the pressure gradient scale $\left(p_{\mathrm{x}}, p_{\mathrm{y}}, p_{\mathrm{z}}\right) \sim \rho_{0} g \beta H_{0} h / k$, and the attenuation coefficient scale $\eta \sim h^{-1}$. The system equations are rewritten in dimensionless form as follows:

$$
\begin{gathered}
u_{t}+u u_{x}+v u_{y}+w u_{z}=-\left(\operatorname{Pr}^{2} G r\right) p_{x}+\operatorname{Pr} \nabla^{2} u, \\
v_{t}+u v_{x}+v v_{y}+w v_{z}=-\left(\operatorname{Pr}^{2} G r\right) p_{y}+\operatorname{Pr} \nabla^{2} v+\left(\operatorname{Pr}^{2} G r\right) \tau, \\
w_{t}+u w_{x}+v w_{y}+w w_{z}=-\left(\operatorname{Pr}^{2} G r\right) p_{z}+\operatorname{Pr} \nabla^{2} w, \\
\tau_{t}+u \tau_{x}+v \tau_{y}+w \tau_{z}=\nabla^{2} \tau+\left(\eta \mathrm{e}^{\eta y}-2\right), \\
u_{x}+v_{y}+w_{z}=0 .
\end{gathered}
$$

All quantities in equations (18)-(22) are now dimensionless. The temperature boundary condition on the sloping bottom now becomes

$$
\begin{aligned}
& \frac{\partial \tau}{\partial n}=-\mathrm{e}^{-A \eta x}+\varepsilon[\operatorname{rand}(0,1)-0.5] \quad \text { (random perturbation), } \\
& \frac{\partial \tau}{\partial n}=-\mathrm{e}^{-A \eta x}+0.5 \varepsilon\left[\sin \left(k_{c} z+\phi\right)\right] \quad \text { (single-mode perturbation). }
\end{aligned}
$$

Other boundary conditions for temperature and velocity remain in the same forms as the dimensional ones.

Equations (18) to (22) are solved using a finite difference method. The velocity components and temperature are directly obtained from the Navier-Stokes equations and the energy equation, and the pressure is calculated from a pressure Poisson equation formed directly from the Navier-Stokes equations. Standard second-order 
central differencing is used for all spatial derivatives except the nonlinear terms in the momentum and energy equations, which are approximated with a modified second-order upwind scheme. The time integration for the velocity components and temperature is by a second-order time-accurate backward differencing scheme. The system equations are solved implicitly with an iterative procedure. Details of the numerical schemes can be found in Lei, Cheng \& Kavanagh (2001).

\section{Preliminary tests}

In the present study, all calculations are carried out in a domain of dimensions corresponding to those of the experimental model described in Lei \& Patterson (2002a), i.e. $L=10, W=5, h=1$ and $A=0.1$ in dimensionless forms. To avoid a singularity at the tip in the numerical calculations, the tip is cut off at $x=1$, and an additional rigid non-slip and adiabatic wall (similar to the wall at the deep end) boundary is assumed here (refer to figure 1). It is anticipated that the flow in the interior of the domain is not modified significantly. The dimensionless bulk attenuation coefficient is also obtained from the experimental measurement, which gives $\eta=0.37$, satisfying the condition of the shallow water assumption. The convective flows are calculated at Prandtl numbers around 7.

Prior to the calculations, preliminary tests with random perturbations are carried out to investigate the dependence of the numerical solutions on the perturbation amplitude, the grid resolution and the time-step. For calculations with random perturbations, the perturbation varies arbitrarily over the entire sloping bottom. The random perturbations are also regenerated at each time step. Therefore, both the distribution and the amplitude of the perturbation vary arbitrarily. All calculations start from $t=0$ with the same initial conditions and proceed until the convective instability is fully established.

\subsection{Selection of the amplitude of perturbation}

We first examine the system response to different perturbation amplitudes to ensure that the selected amplitude for this study is within the range in which the system response is linear. Three different values of the amplitude $(\varepsilon=0.5 \%, 1.0 \%$ and $2.0 \%$ respectively) are calculated for the case of $\operatorname{Pr}=6.83$ and $G r=2.51 \times 10^{6}$, corresponding to the experimental parameters reported in Lei \& Patterson (2002a). As noted previously, both the experimental observations and corresponding numerical simulations (Lei \& Patterson 2001) have revealed three stages of the flow development in this case from an isothermal and stationary state: an initial stage, a transitional stage and a quasi-steady stage. The present study is mostly concerned with the flow development at the initial and transitional stages.

The calculated results with different amplitudes of the perturbation source are presented in figures $2(a)$ and $2(b)$ and table 1. Figure 2(a) plots the typical temperature profiles along the transverse direction at a time after the instability is well established $(t=0.01)$. These plots are for a position on the sloping bottom and near the centre of the enclosure $(x=5.5$ and $y=-0.55)$. Similar results are obtained for other locations, and the dependence of the results on the horizontal location will be discussed in $\S 4.2$. Clearly, these plots show a wave pattern in the transverse direction. Due to the irregular nature of the occurrence of the rising plumes and the random perturbation, the transverse waveforms exhibit irregularities. However, it is clear in this figure that there is a dominant wavelength/wavenumber in the transverse direction, which is the same for all three plots (see table 1). It is also clear in figure 2(a) that different 

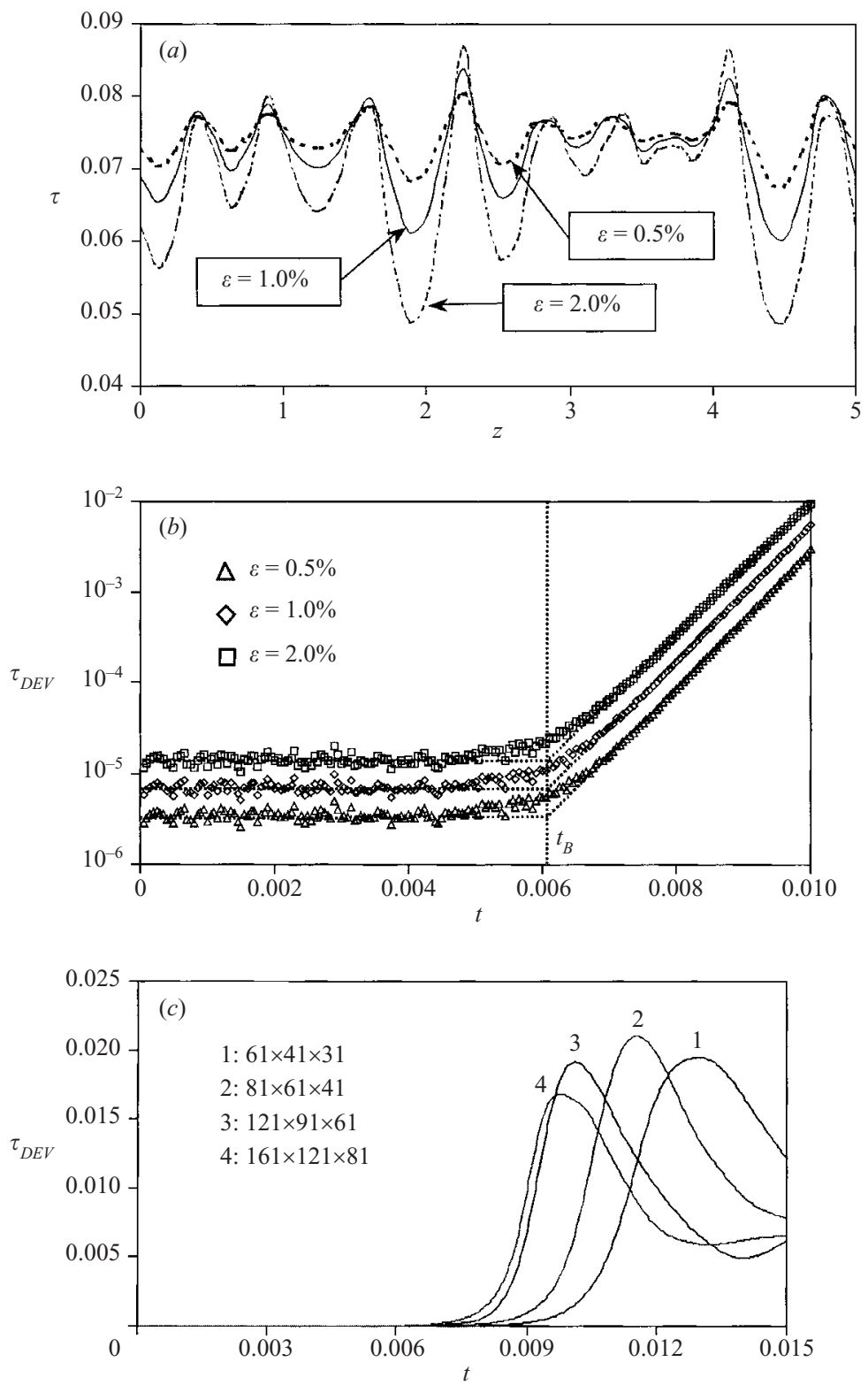

FIGURE 2. Preliminary tests with $(a)$ and $(b)$ showing the flow responses to different amplitudes of the perturbation source and $(c)$ showing the grid-dependence of the response. Data plotted here are for the position $(x, y)=(5.5,-0.55)$. (a) Typical transverse temperature profiles with the presence of the convective instability $(t=0.01)$. $(b),(c)$ Growth of the standard deviation of the temperature in the transverse direction.

amplitudes of the perturbation source result in different amplitudes of the temperature response, which can be measured by the standard deviation of the temperature plots. The calculated standard deviation for the time and location considered are listed in table 1 , indicating a linear increase with the perturbation amplitude.

In fact, the system response to perturbations, or the growth of perturbations, is indicated well by time series of the standard deviation of temperature along the 


\section{Responses}

Dominant wavenumber at $t=0.01$

Standard deviation at $t=0.01$

Amplitude of response $(a)$

Predicted time for the onset of instability $\left(t_{B}\right)$

Growth rate of the standard deviation in the exponential-growth region $(c)$

$\begin{array}{ccc}\varepsilon=0.5 \% & \varepsilon=1.0 \% & \varepsilon=2.0 \% \\ 10.2 & 10.2 & 10.2 \\ 0.0028 & 0.0053 & 0.0097 \\ 3.5 \times 10^{-6} & 7.0 \times 10^{-6} & 1.4 \times 10^{-5} \\ 0.00615 & 0.00613 & 0.00610 \\ 1735.4 & 1715.7 & 1678.8\end{array}$

TABLE 1. System responses to different amplitudes of the perturbation source. Data presented here are obtained from the transverse temperature profiles at the position $(x, y)=(5.5,-0.55)$.

transverse direction, which can be found in figure $2(b)$. Note that the standard deviation is plotted on a logarithmic scale in this figure. Clearly, each of the plots in figure $2(b)$ can be divided into three regions in time: a constant-response region, an exponential-growth region (represented by the linearly increasing part of the curve) and a transitional region connecting the above two regions. It must be recognized that these three time regions are different from the three transient stages of the flow development mentioned earlier. Although the constant-response region approximately corresponds to the aforementioned initial stage of the flow development, the transitional and exponential-growth regions shown in figure $2(b)$ both lie within the transitional stage of the overall flow development. In the constant-response region, the perturbation is not amplified, suggesting that the flow, and in particular the bottom thermal boundary layer, is stable, and the system response echoes the random perturbation. The time-averaged standard deviation in the constant-response region, known as the amplitude of response $(a)$ in table 1 , also indicates a linear dependence on the perturbation amplitude.

In the exponential-growth region, the perturbation grows exponentially in time, which is represented by

$$
\tau_{D E V}=a \exp \left(c\left(t-t_{B}\right)\right)
$$

where $\tau_{D E V}$ is the standard deviation of temperature along the transverse direction, $a$ is the amplitude, $c$ is the growth rate and $t_{B}$ is the critical time for the onset of three-dimensional instability. The growth rate $c$ then corresponds to the slope of the linearly increasing part in figure $2(b)$, which can be determined accordingly. The results are given in table 1 . It is seen in table 1 that there is a slight variation of the growth rate with the amplitude of the perturbation source, and the growth rate for the smaller amplitude is slightly higher than that for the larger amplitude. The maximum variation of the growth rate is about $3.0 \%$ for the parameters examined here. This variation is insignificant relative to the variation of the amplitude of perturbation, and thus the growth rate can be treated as a constant. Since there is no definite criterion for determining the critical time $t_{B}$ for the onset of the instability, it is approximately determined in figure $2(b)$ as the intersection point between the constant-response curve and the exponential-growth curve. The results obtained using this method are listed in table 1 . It is seen that the critical time is independent of the amplitude of the perturbation source.

In summary, within the range of parameters examined here, the variation of the perturbation amplitude does not change the stability properties of the flow (e.g. the critical time for the onset of instability, the dominant wavenumber and the growth rate of perturbations), and the system response to perturbations is linear. Subsequent calculations will be conducted with a fixed perturbation amplitude of $\varepsilon=1.0 \%$. 


\begin{tabular}{ccccc}
\hline$\quad$ Mesh number & 1 & 2 & 3 & 4 \\
$\quad$ Mesh size & $61 \times 46 \times 31$ & $81 \times 61 \times 41$ & $121 \times 91 \times 61$ & $161 \times 121 \times 81$ \\
$\begin{array}{c}\text { Predicted time for the onset } \\
\text { of instability }\end{array}$ & 0.006842 & 0.006130 & 0.005394 & 0.005134 \\
$\begin{array}{c}\text { Growth rate of the standard } \\
\text { deviation }\end{array}$ & 1602.7 & 1715.7 & 1873.1 & 1915.3 \\
Peak time in the growth curve & 0.0130 & 0.0116 & 0.0101 & 0.0098
\end{tabular}

TABLE 2. Results of the mesh-dependence test. Data presented here are obtained from the transverse temperature profiles at the position $(x, y)=(5.5,-0.55)$.

\subsection{Dependence on grid resolution and time-step}

The above calculations are all conducted on an $81 \times 61 \times 41$ mesh (Mesh 2) with 81 nodes over the length $(L), 61$ nodes over the depth $(h)$ and 41 nodes over the width $(W)$. Grid stretching is used along the length and depth to concentrate nodes in the regions near all boundaries. In the region near the sloping bottom, the mesh is stretched such that there are 15 cells located within the bottom thermal boundary layer (based on the experimental measurement of Lei \& Patterson 2002a). In the transverse direction, the mesh is evenly distributed along the width. The time-step is fixed at $\Delta t=10^{-6}$. Previous studies have shown that the major features of the transient flow development observed in the experiment can be reproduced in both two-dimensional and three-dimensional simulations with this grid and time-step (Lei $\&$ Patterson 2001). Additional grid dependence tests have been carried out for the present stability problem by obtaining results on a coarser mesh (Mesh 1: $61 \times 46 \times 31$ ) and two finer meshes (Mesh 3: $121 \times 91 \times 61$ and Mesh 4: $161 \times 121 \times 81)$ respectively. The results are compared in figure $2(c)$ and table 2 .

Figure 2(c) plots the growth curves of the standard deviation of the temperature along the straight line of $(x, y)=(5.5,-0.55)$ obtained with all four meshes. Clearly, the predicted growth of the perturbation depends strongly on the grid resolution. The following three major quantities of the growth curve are affected by the grid resolution: the predicted time for the onset of instability; the growth rate of the perturbation in the exponential growth region; and the peak time of the growth curve. Quantitative comparisons of these quantities are given in table 2. It is seen that the predicted values of all the three quantities vary significantly from the coarsest mesh (Mesh 1) to the finest mesh (Mesh 4). The variations for the onset time, growth rate and peak time are $33.3 \%, 16.3 \%$ and $32.8 \%$ respectively. However, the variations between the second finest mesh (Mesh 3) and the finest mesh (Mesh 4) for all quantities are within $5 \%$. Since the basic features of the flow can be produced even on the second coarsest mesh (Mesh 2), Mesh 3 can provide adequate resolution for the present analysis.

In what follows, the results are all obtained with the $121 \times 91 \times 61$ mesh unless specified. The time-step is fixed at $\Delta t=10^{-6}$ for all calculations.

\section{Results and discussion}

\subsection{Flow development}

Consider the case of $\mathrm{Pr}=6.83$ and $\mathrm{Gr}=2.51 \times 10^{6}$. The detail of the flow development for this case from an isothermal and stationary state has been examined numerically (Lei \& Patterson 2001) and in conjunction with a model experiment 


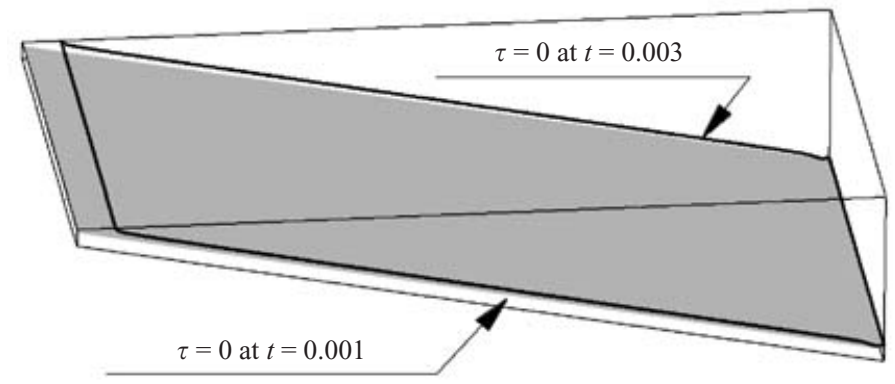

FIGURE 3. Temperature structures at the early stage. Plotted are the temperature iso-surfaces of $\tau=0$ at $t=0.001$ and 0.003 respectively.

(Lei \& Patterson 2002a). These investigations revealed the three distinct stages of the flow development referred to above, an early stage, a transitional stage and a quasi-steady stage. The previous numerical observations were based on unperturbed solutions, whereas the present analysis is based on perturbed solutions. The indication from the perturbation sensitivity tests was that the perturbation does not alter the flow structures at the initial stage. It is also anticipated that the perturbed solution will eventually result in the same quasi-steady flow as the unperturbed solution. However, different flow structures are observed at the early transitional stage. Therefore, emphasis here will be placed on the flow structures at the early transitional stage, whereas the major flow features at other stages will be briefly summarized.

\subsubsection{Early stage flow}

The early-stage flow is characterized by a steady growth of a thermal boundary layer along the sloping bottom. This is demonstrated in figure 3 , which plots the temperature iso-surfaces $(\tau=0)$ at two different times. The temperature value is chosen so that the iso-surface approximately represents the boundary between the thermal boundary layer and the core region. Therefore, the increase of the distance between the iso-surface and the sloping bottom indicates the growth of the thermal boundary layer (see figure 3). It is observed in the numerical simulation that the temperature variation in the core region is negligible compared with that within the thermal boundary layer, suggesting that the bottom heating dominates the radiation absorption by the water column at the early stage. Figure 3 also shows that the temperature structure is purely two-dimensional at this stage. Furthermore, it is seen in this figure that the iso-surfaces of $\tau=0$ are approximately parallel to the sloping bottom, indicating that the thermal boundary layer is parallel to the bottom. Outside the thermal boundary layer, the temperature iso-surfaces are parallel to the water surface, which represents a weak stratification due to direct absorption of the radiation by the water body.

While the thermal boundary layer grows, a distinct horizontal temperature gradient as well as a vertically adverse temperature gradient develops within the thermal boundary layer. The horizontal temperature gradient then initiates a flow up the slope. In the region near the tip, the boundary layer flow discharges into the core region, and thus an upper intrusion flow travelling from the shallow end to the deep end is formed. As a consequence, a clockwise circulation is established in the enclosure. The flow structure at this stage shows a single cell with closed streamlines. 

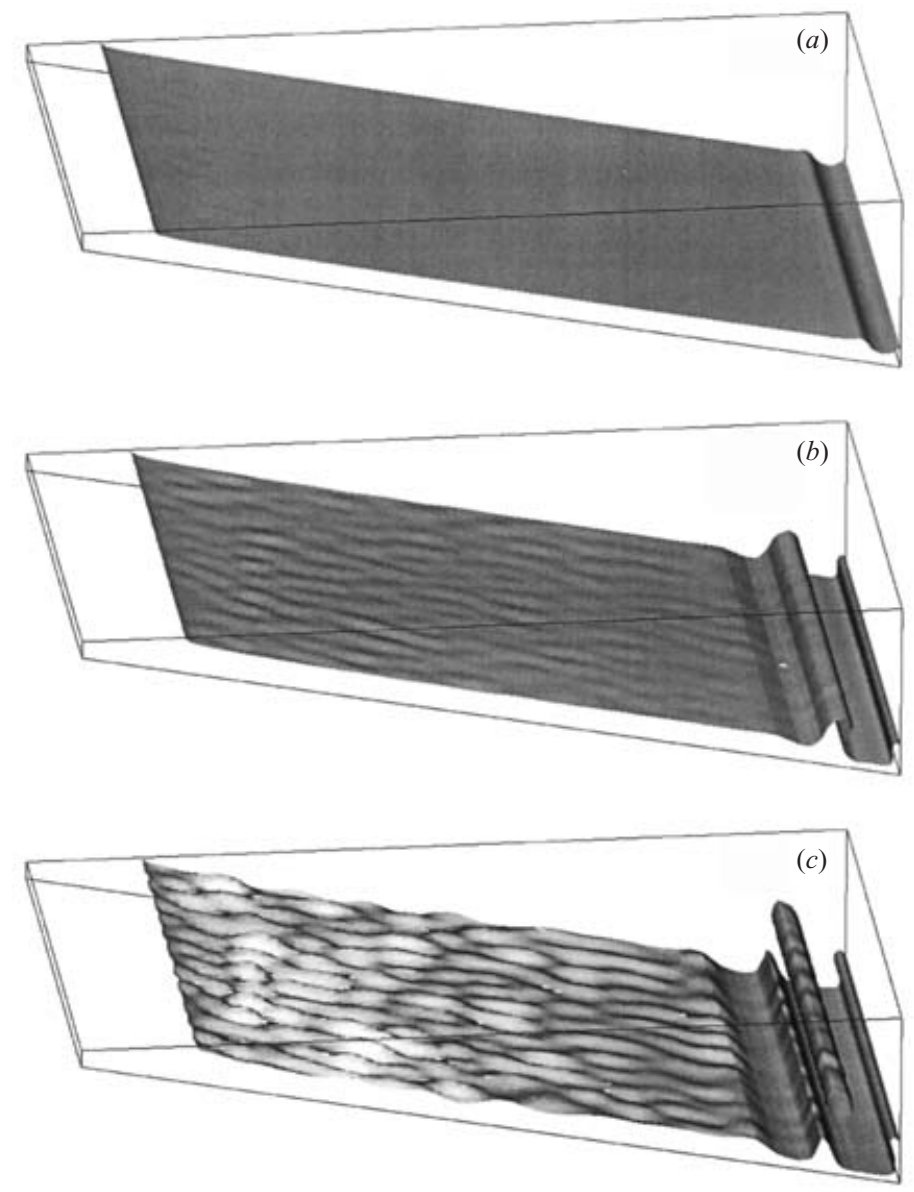

FIGURE $4(a-c)$. For caption see facing page.

\subsubsection{Transitional stage flow}

The flow structures at the transitional stage are shown in figure 4, in which figures $4(a)-4(e)$ plot the temperature iso-surfaces and figure $4(f)$ plots the isosurface of the streamwise vorticity. The streamwise vorticity, $\omega_{x}$, is defined as

$$
\omega_{x}=\frac{\partial v}{\partial z}-\frac{\partial w}{\partial y} .
$$

For the present flow parameters, two different types of flow instabilities, a twodimensional flow instability and a three-dimensional Bénard-type instability, are observed from the three-dimensional calculation at the transitional stage. The twodimensional flow instability, which is in the form of hydraulic-jump-like structure, starts from the very deep corner (see figure $4 a$ ). This instability is evidently triggered by a two-dimensional perturbation due to the discontinuity of the thermal boundary conditions at this corner. Other mechanisms such as the ejection of fluid from the vertical boundary layer in response to the general circulation in the domain may be possible but are unlikely to be strong enough to generate the jump like structure. As 

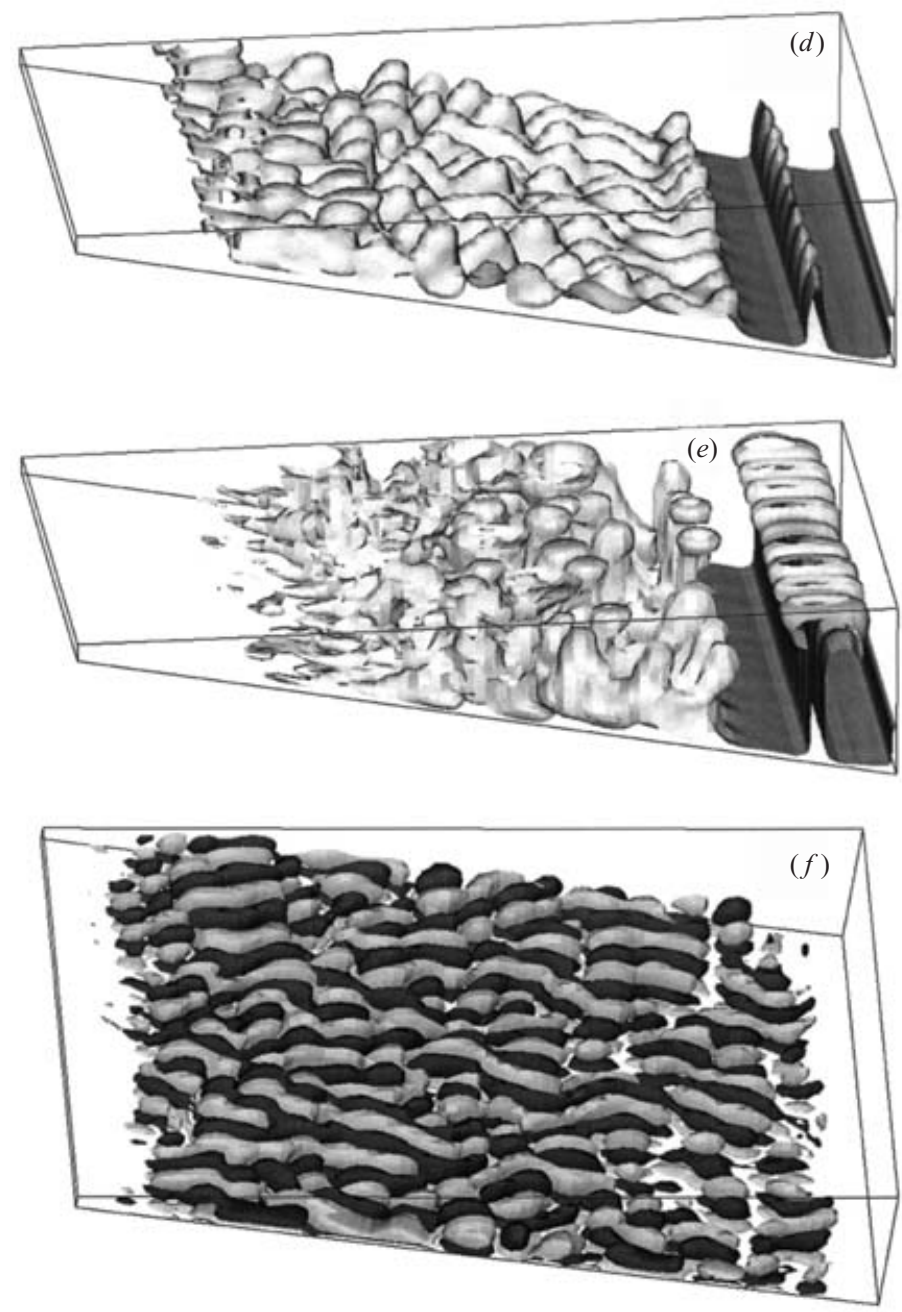

FIGURE 4. Flow structures at the early transitional stage. $(a-e)$ Temperature iso-surfaces at (a) $t=0.005$, (b) $t=0.007,(c) t=0.008,(d) t=0.009$ and $(e) t=0.010$. ( $f$ ) Iso-surface of the streamwise vorticity at $t=0.006$.

time passes, the jump-like structure amplifies and moves downstream (up the slope, see figures $4 a, 4 b)$. As a consequence of the amplification, the thermal boundary layer is thickened locally. At a certain stage, the jump-like structure itself becomes unstable to the Bénard-type instability, and thus is released from the thermal boundary layer into the upper water layer (see figure 4c). In the unperturbed case, it was observed that the two-dimensionality is retained until after the release of the jump-like structure (Lei \& Patterson 2001).

The present analysis is mostly concerned with the three-dimensional convective instability caused by the adverse temperature gradient within the thermal boundary layer due to the bottom heating. Previous scaling analysis has shown that the stability feature of the thermal boundary layer is governed by a local Rayleigh number which is a function of the thickness of this layer (Lei \& Patterson 2002b). The three-dimensional convective instability must be triggered by three-dimensional perturbations. In the 
unperturbed case, the three-dimensional perturbation comes from the accumulation of numerical errors. Since truncation errors in numerical simulations are normally largest near boundaries, the three-dimensional instability is seen to develop starting from corners between the sidewalls and the top and bottom boundaries in the unperturbed case (Lei \& Patterson 2001). However, with the artificial perturbation in place, the situation is now different. It is observed in the present simulation that the three-dimensional instability simultaneously develops everywhere across the thermal boundary layer.

For the case under consideration, the thermal boundary layer becomes unstable at a time around $t=0.005$ but the convective instability becomes visible only after $t=0.006$ (see figures $4 b-4 e$ ). As the surface heating continues, the instability becomes increasingly strong and eventually manifests itself in the form shown. Figure 4 also indicates that there is a transition from one unstable mode to another. At the early stage after the onset of the instability, convective rolls with axes approximately aligned with the slope are observed (figures $4 b$ and $4 c$ ). This flow structure is also confirmed in the plot of the iso-surfaces of the streamwise vorticity (figure $4 f$ ). In this plot, the light and dark patterns represent negative and positive vorticity respectively. Clearly, a distinct dominant transverse wavelength is present at this stage. The convective rolls are the preferred mode at the onset of the Rayleigh-Bénard instability in an inclined layer of low Rayleigh numbers (Kurzweg 1970; Foster 1971). This convective mode is short-lived in the present case due to the excessive bottom heating which quickly raises the local Rayleigh number far beyond the critical value. It is seen in figures $4(c)$ and $4(d)$ that streamwise wave patterns are developing on top of the longitudinal rolls. As time passes, the superimposed convective patterns evolve into another mode of the convective instability, that is plumes which contain warm water convecting upward from the thermal boundary layer (figure $4 e$ ). At this time, the global flow seems to be 'chaotic' to a certain extent. However, careful examination of the flow structure near the sloping bottom indicates that regularity, or more specifically a regular transverse wavelength, is retained within the thermal boundary layer following the onset of the three-dimensional convective instability (§4.2).

\subsubsection{Quasi-steady flow}

In the present case, heat is conducted into the lower layer of the water body from the sloping bottom, and convected away from the boundary layer by the primary largescale circulation as well as the secondary convection. Both the primary and secondary convections result in a significant increase of the temperature in the upper water layer. At a certain stage, the heat conducted into the thermal boundary layer is balanced by that convected away from it, and thus the growth of the thermal boundary layer ceases. A quasi-steady state is reached when the temperature increases at the same rate everywhere in the domain. The typical temperature structure at the quasi-steady state obtained for the present parameters is shown in figure 5 . The result plotted in this figure was obtained on the $81 \times 61 \times 41$ mesh without artificial perturbation. A similar result is expected for the perturbed case on finer meshes. Due to the enormous amount of CPU time required for the finer meshes, the present calculations were terminated before a quasi-steady stage was reached.

At the quasi-steady stage, a three-layer temperature structure, with an upper layer, a middle layer and a lower layer, is established in the wedge. In the upper layer, a stabilizing stratification exists, and in the lower layer (boundary layer), a destabilizing stratification is present. The middle layer connects the upper and lower layers (Lei \& Patterson 2001). It is found that a distinct horizontal temperature gradient is 


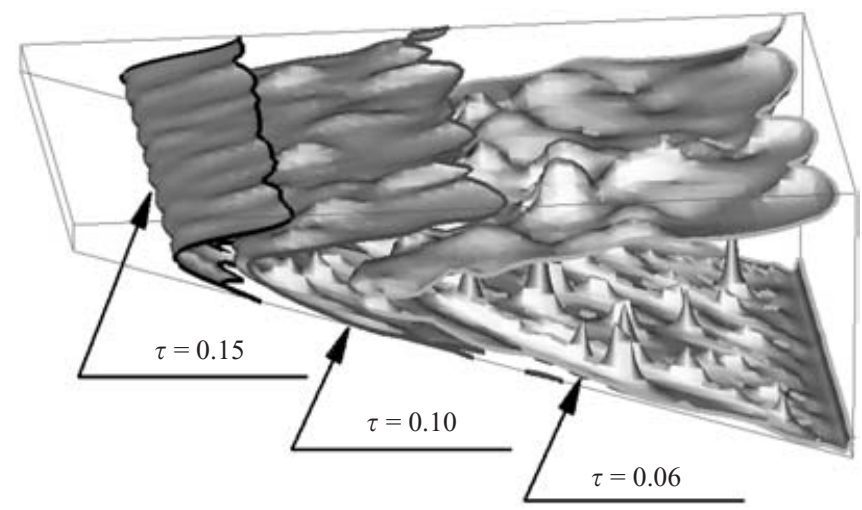

Figure 5. Temperature structures at the quasi-steady stage. Plotted are the temperature iso-surfaces of $\tau=0.06,0.10$ and 0.15 at $t=0.2$. The result was obtained on the $81 \times 61 \times 41$ mesh without artificial perturbation.

established in the upper water layer in addition to the horizontal temperature gradient in the thermal boundary layer. Both horizontal temperature gradients are responsible for maintaining the steady large-scale circulation across the domain, whereas the vertical adverse temperature gradient in the thermal boundary layer also maintains a weak secondary motion at the quasi-steady state.

\subsection{Flow response to random perturbations}

First we examine the flow response to random perturbations at fixed parameters of $\operatorname{Pr}=6.83$ and $G r=2.51 \times 10^{6}$ as considered above, and we focus on the dependence of the flow response on the horizontal location. A growth curve similar to those presented in figures $2(b)$ and $2(c)$ can be obtained for any location along the thermal boundary layer, from which quantitative information such as the critical time and growth rate can be extracted.

Figure 6(a) plots the predicted critical time for the onset of the convective instability against the horizontal location. It is clear that the flow is more stable at both the very shallow and deep ends (the convective instability sets in at a later time). At the shallow end, the boundary layer flow changes direction to form an upper intrusion flow, and the isotherms become almost vertical. Therefore, the adverse temperature gradient over the thermal boundary layer is reduced, which in turn stabilizes the flow. In this region, the conduction effect is significant. At the deep end, the relatively high stability is evidently caused by the compression due to the two-dimensional instability. As noted earlier, the inconsistent thermal conditions at the bottom and endwall generates a jump-like structure which thins parts of the boundary layer and thickens others. However, at early times (before the majority of the thermal boundary layer becomes unstable), the compression is distinct, whereas the thickening is hardly noticeable. This can be seen in figure $4(a)$ and from the temperature structures at earlier times. Previous scaling has shown that the stability of the thermal boundary layer depends on a local Rayleigh number, which is a function of the thickness of the thermal boundary layer (Lei \& Patterson 2002b). Therefore, the compression results in a reduction of the thickness, which in turn reduces the local Rayleigh number and stabilizes the local boundary layer.

Apart from these two end regions, the majority of the thermal boundary layer becomes unstable at about the same time (approximately at $t=0.00525$, averaged 

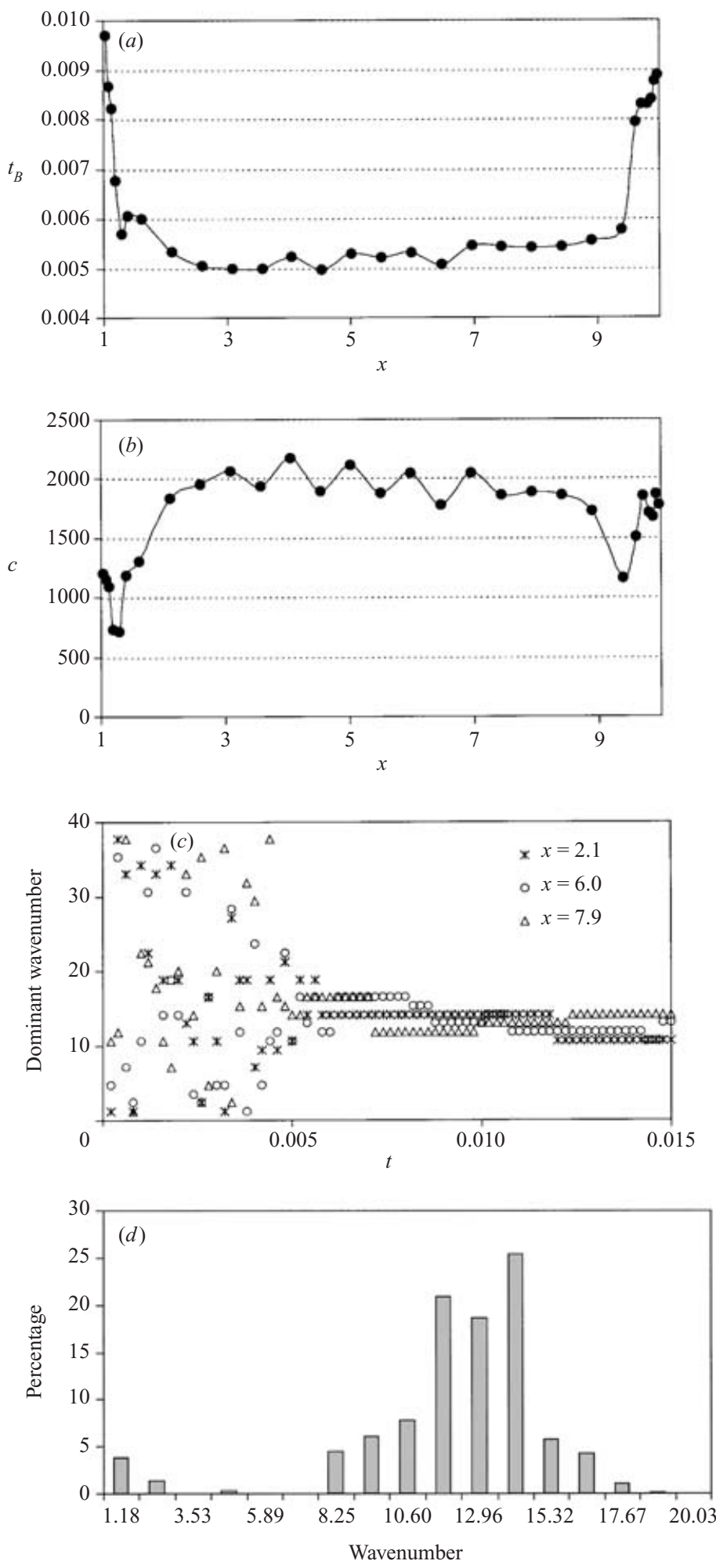

FIGURE 6. For caption see facing page. 
value for central locations). This confirms that the thermal boundary layer grows almost uniformly along the sloping bottom. Further examination of the data plotted in figure 6(a) shows that, within the central part of the thermal boundary layer, there is a slight increase of the critical time from the shallow region to the deep region despite the zigzag behaviour of the plot. The variation of the critical time is about $8 \%$, which is due to the non-uniform heat flux condition applied to the slope. The zigzag behaviour of the critical time at the central locations is likely to be associated with the streamwise wavelength.

In figure $6(b)$ the growth rate of the perturbation is plotted against the horizontal location. It is seen in this figure that the growth rate is lower near the shallow and deep ends, which again indicates that the flow is more stable toward both ends. In fact, for most of the locations near the shallow and deep ends, the growth of the perturbation is not fully exponential due to the nonlinear effects noted above. Nevertheless, the data shown in figure $6(b)$ are fitted with the assumption that at all locations the perturbation grows exponentially. Therefore, the growth rates given in figure $6(b)$ for positions near both ends may contain large errors. In the following discussion, we will concentrate on the growth of the central part of the thermal boundary layer (approximately from $x \approx 2.0$ to $x \approx 9.0$ ).

In that region, the growth rate takes a value around 2000. Similar to the plot of the critical time, the distinct zigzag behaviour of the growth rate in figure $6(b)$ is also likely to be associated with the streamwise wavelength. Figure $6(b)$ also shows that there is a slight decrease of the growth rate from the shallow region to the deep region, which is again attributed to the inhomogeneous heat flux condition on the slope.

As noted previously, a particular transverse wavelength/wavenumber is selected after the onset of the three-dimensional convective instability. This is demonstrated for three selected locations in figure 6(c). Plotted here are the time series of the dominant transverse wavenumbers at each location, which are obtained by taking the fast Fourier transform (FFT) of temperature signals. This figure shows that the dominant wavenumber is arbitrary before the onset of the convective instability (prior to $t \approx 0.00525$ ), echoing the random perturbation. However, it becomes very regular and approximately the same at each location after the onset of the instability. Similar plots are obtained for other locations along the thermal boundary layer. None of these results indicates a clear dependence of the dominant wavenumber on the location along the length.

Figure $6(d)$ plots the distribution of the dominant wavenumber after the onset of the instability (from $t=0.00525$ to $0.015,196$ recorded times). Plotted in this figure are the statistical data for all available central locations (from $x \approx 2.0$ to 9.0, 15 recorded locations). The total number of samples is about 3000. It is clear that most of the dominant wavenumbers are within the range from 8 to 18 with a clear dominance between 11 and 15 . Figure $6(d)$ also shows that only about $5 \%$ of the sampled wavenumbers lie outside the range from 8 to 18 . Within this $5 \%$, nearly $4 \%$ take a value of 1.18 , which is associated with the periodic boundary conditions applied to the transverse direction.

FIGURE 6. Flow response to a random perturbation at $G r=2.51 \times 10^{6}$ and $\operatorname{Pr}=6.83$. (a) Critical time at the onset of convective instability. (b) Growth rate of the perturbation. (c) Typical time series of dominant wavenumbers. $(d)$ Distribution of the dominant wavenumbers after onset of instability. 
In order to determine the preferred mode of the convective instability for particular flow conditions, the flow response to single-mode perturbations is investigated next.

\subsection{Flow response to single-mode perturbations}

In this section, the preferred mode of the convective instability in the thermal boundary layer is determined for the case of $P r=6.83$ and $G r=2.51 \times 10^{6}$. The same procedures can be applied to any other flow conditions. Instead of the random perturbation, single-mode perturbations with a series of wavenumbers are applied to the heat flux condition on the sloping bottom. Since the present study is mostly concerned with the transverse wavelength/wavenumber, the single-mode perturbation is only applied along the transverse direction (i.e. the perturbation is in the form of a transverse wave). In this study, nine different wavenumbers within the range of 8 to 20 are calculated. The results are presented in figure 7.

Figure 7(a) plots the calculated critical times at the onset of the convective instability against wavenumber for three central locations. The variation along the horizontal locations, as noted previously, is clearly seen in this plot. The dependence of the critical time on the transverse wavenumber is also obvious in figure 7( $a)$. It is seen that, for all three locations, the value of the critical time is smallest around the wavenumbers 14 to 15 . This is also true for other central locations (see figure $7 b$ ), suggesting that the most unstable mode of the convective instability has a transverse wavenumber around 14 to 15 . It is also clear in figure $7(b)$ that, for all single-mode perturbations investigated, the critical time is smallest around $x=3.0$, which is the most unstable region.

The calculated growth rates of the perturbation for all modes are presented in figures $7(c)$ and $7(d)$. Figure $7(c)$ plots the growth rate against the wavenumber, and figure $7(d)$ plots the growth rate against the horizontal position. Again, the dependence of the growth rate on the horizontal position is clearly seen in these plots, which is consistent with the observations for random perturbations. Both figures demonstrate that the growth rate of the perturbation is largest around the wavenumber 12 to 13 , indicating that the most amplified mode of the convective instability has a transverse wavenumber between 12 and 13. Figure $7(d)$ also confirms that the most unstable region lies around $x=3.0$, where the largest amplification is present with all single-mode perturbations.

It is worth noting that in the above discussion the most unstable mode is identified based on the comparisons of the starting time of the three-dimensional instability, which represents a single point in time. As illustrated in figure $2(b)$, the determination of the critical time contains some uncertainty due to the transitional features around this point. Moreover, the most amplified mode is identified as the mode with the largest growth rate. In general, we would expect the growth rate of the perturbation to be a function of time. However, the growth rate given in this study represents an averaged quantity over a period of time (for $t>t_{B}$, see figure $2 b$ ). Therefore, it is not surprising to see that the most unstable mode, identified based on a single point in time, is different from the most amplified mode, which is averaged over a period of

FIGURE 7. Flow response to single-mode perturbation at $G r=2.51 \times 10^{6}$ and $\operatorname{Pr}=6.83$. (a) Critical time versus wavenumber at selected locations. (b) Critical time versus horizontal position for all modes. $(c)$ Growth rate versus wavenumber at selected locations. $(d)$ Growth rate versus horizontal position for all modes. 

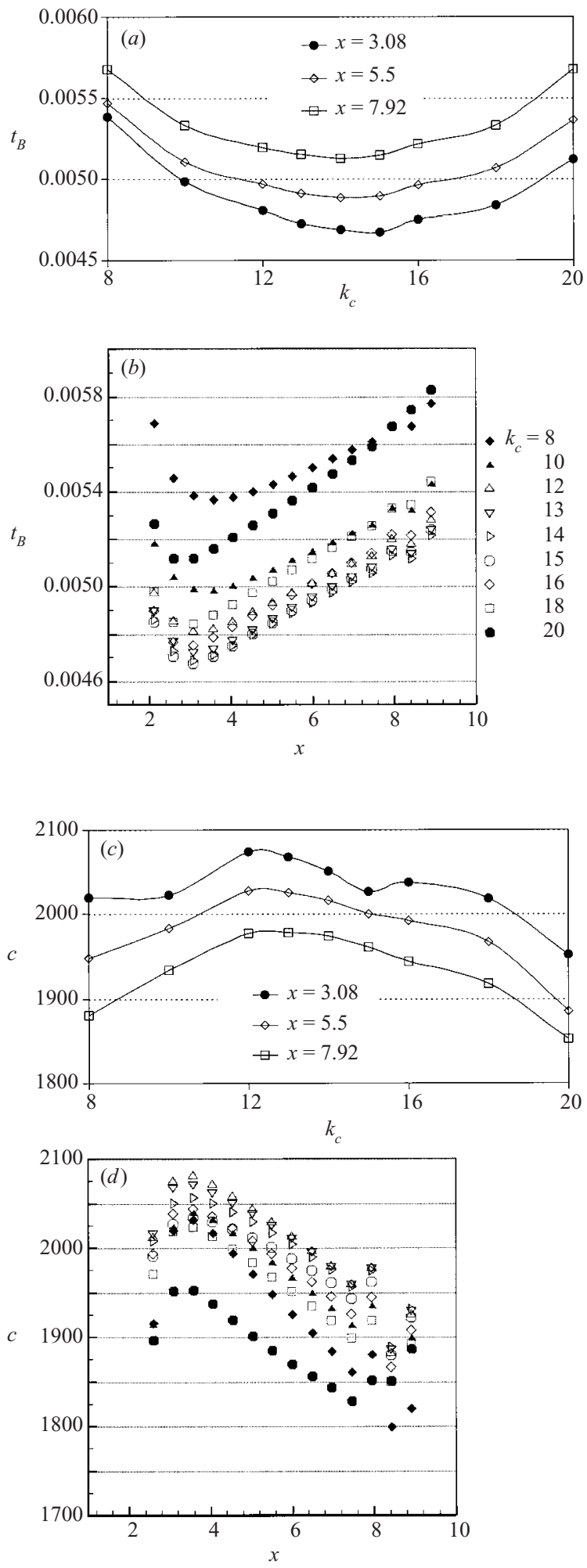

FIGURE 7. For caption see facing page. 

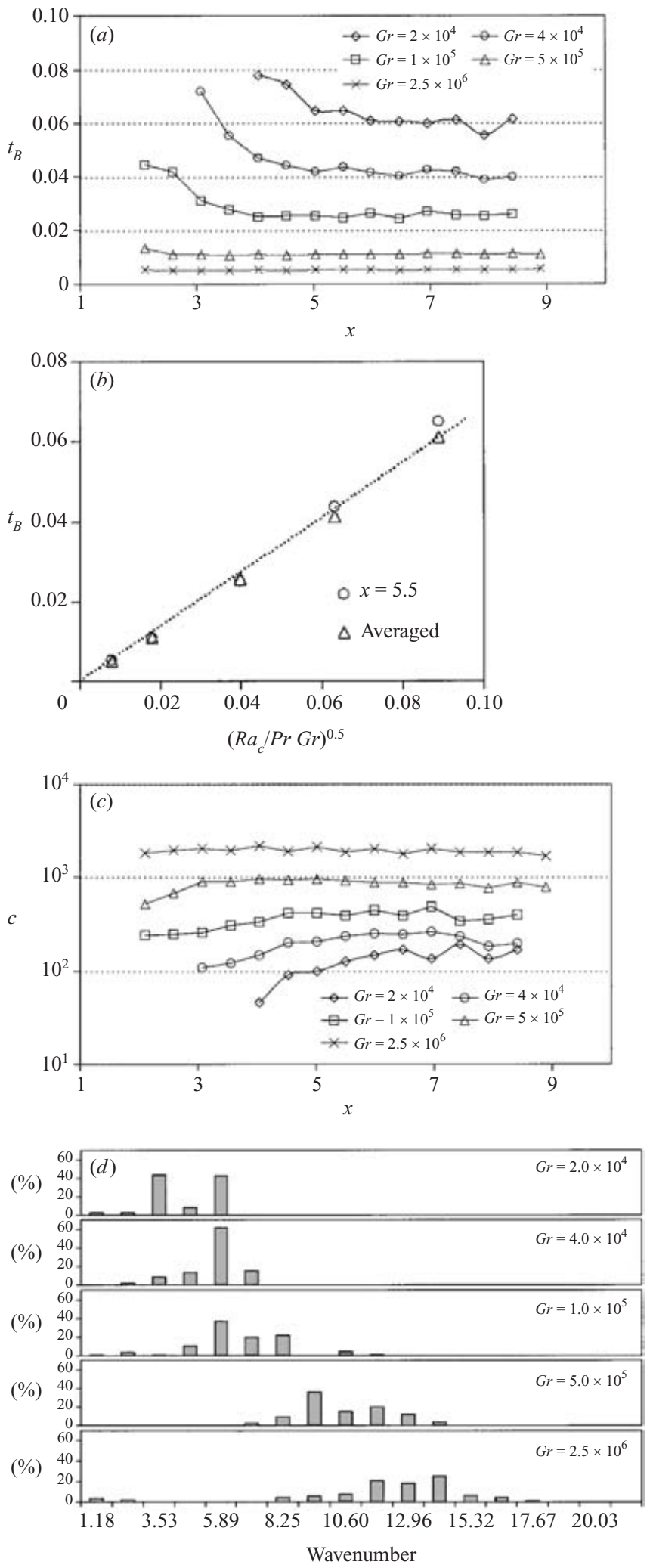

FIGURE 8. For caption see facing page. 
time. Nevertheless, these two modes are found to be reasonably close based on the present analysis.

\subsection{Grashof number effects}

We now consider the flow response to the random perturbation at different Grashof numbers. For this study, the Prandtl number is fixed at 7 . Results are obtained for different Grashof numbers ranging from $10^{3}$ to $2.5 \times 10^{6}$, and are presented in figure 8. Before discussing the results for different Grashof numbers, it is useful to briefly review the outcome of an earlier scaling analysis (Lei \& Patterson 2002b) that is closely relevant to the present investigation.

The scaling analysis for shallow wedges $(h \eta<1)$ with small bottom slopes $(A \ll 1)$ reveals that the heat conduction from the sloping bottom into the water body results in a thermal boundary layer growing along the slope on the scale of $\delta \sim t^{1 / 2}$. The heat is then convected away at a velocity of $u \sim(A P r G r) t^{2}$. In the meantime, the RayleighBénard instability develops in the thermal boundary layer due to the existence of a vertical adverse temperature gradient. The stability characteristics of the thermal boundary layer are quantified by a local Rayleigh number, which is a function of the global Rayleigh number ( $P r G r$, see equations (1) and (2) for definitions) and the thickness of the thermal boundary layer. Depending on the global Rayleigh number and the bottom slope, different flow regimes are possible. For a typical situation with $R a_{c}>A^{-2}$, the flow can be classified as either conductive or transitional or convective. In both the conductive and transitional regimes, the thermal boundary layer is stable to the Rayleigh-Bénard instability, whereas in the convective regime, the thermal boundary layer is unstable. The critical Rayleigh number scale, which separates the stable and unstable regimes, is found to be

$$
(\operatorname{Pr} G r)_{\text {critical }} \sim A^{4} \mathrm{Ra}_{c}^{3} ;
$$

and for $\operatorname{Pr} G r>A^{4} R a_{c}^{3}$, the convective instability sets in at a time scale

$$
t_{B} \sim\left(\frac{R a_{c}}{\operatorname{Pr} G r}\right)^{1 / 2} .
$$

Applying the scaling results directly to the present case gives a critical Rayleigh number of $1.35 \times 10^{5}$ for a bottom slope of $A=0.1$. Given that the Prandtl number is fixed at 7, the corresponding critical Grashof number is then $1.93 \times 10^{4}$. In the present direct stability analysis, no growth of the perturbation is detected at the Grashof number $10^{3}$, suggesting that the thermal boundary layer is stable to the Rayleigh-Bénard instability. At $G r=5 \times 10^{3}$, an extremely weak growth of the perturbation is detected at locations associated with the passage of the twodimensional instability (hydraulic-jump-like structure, see $\S 4.1 .2$ ). The weak growth is due to the local thickening of the thermal boundary layer caused by the twodimensional instability. Apart from this effect, the rest of the thermal boundary layer remains stable to the Rayleigh-Bénard instability. As the Grashof number is increased beyond $5 \times 10^{3}$, relatively stronger growth of the perturbation is detected for a broad range of central locations. The unstable region lies between $x \approx 4.0$ and

FIGURE 8. Flow response to random perturbation at different Grashof numbers. (a) Critical time at the onset of convective instability. $(b)$ Comparison of the calculated critical time with the predicted critical time of the previous scaling analysis. (c) Growth rate of the perturbation. (d) Distribution of the dominant wavenumbers after onset of instability. 
$x \approx 8.5$ at $G r=10^{4}$, and it expands toward both the shallow and deep ends as the Grashof number is further increased. However, the three-dimensionality of the flow is difficult to visualize for Grashof numbers below $10^{4}$ owing to its weakness. For Grashof numbers above $2 \times 10^{4}$, the three-dimensionality of the flow becomes clear and increasingly strong as the Grashof number increases. The above observations indicate that the critical Grashof number for the present configuration lies between $5 \times 10^{3}$ and $10^{4}$, consistent with the previous scaling analysis.

For all Grashof numbers above the critical value, the critical time at the onset of the convective instability, the growth rate of the perturbation and other instability properties can be obtained using the same procedures as outlined previously. The results are presented in figure 8 . Figure $8(a)$ plots the calculated critical time against the horizontal location for different Grashof numbers. It is clear in this figure that the critical time increases as the Grashof number decreases. It is also seen that the unstable region shrinks as the Grashof number decreases.

The critical time obtained from the present direct stability analysis is compared with that predicted by the scaling analysis (Lei \& Patterson 2002b) in figure $8(b)$. Here, the horizontal axis is the scaling prediction, and the vertical axis is the calculated data. Plotted here are the critical times at a selected central location and the averaged values for all central locations. Clearly, a linear dependence on the scaling prediction exists for both sets of data. The slopes of the linear fits for these two sets of data are 0.74 and 0.69 respectively. This result confirms the scaling prediction.

The growth rates of the perturbation calculated for different Grashof numbers are presented in figure $8(c)$. This figure clearly demonstrates that the growth rate decreases with the Grashof number. A zero growth rate is expected for Grashof numbers below the critical value, representing a stable flow condition.

Figure $8(d)$ plots the distribution of the dominant wavenumbers obtained at different Grashof numbers. These are the statistical data obtained for central locations where instability is clearly present. The previous numerical experiments for the case with Grashof number $G r=2.51 \times 10^{6}$ suggest that the distribution map approximately indicates the most unstable and amplified modes of the convective instability. It is seen in figure $8(d)$ that the dominant wavenumber shifts toward the lower end as the Grashof number decreases. Figure $8(d)$ also indicates that the spectrum of the instability modes narrows with the decrease of the Grashof number. It is expected that, as the Grashof number approaches the critical value, the numerically obtained dominant wavenumber converges to 1.18 , which is the wavenumber associated with the periodic boundary conditions prescribed on the transverse boundaries.

\section{Conclusions}

The stability properties of the thermal boundary layer developing along the sloping bottom of a water-filled shallow wedge subject to solar radiation are determined by means of direct stability analysis. Both a random perturbation (white noise) and a single-mode perturbation (transverse wave) are applied to the three-dimensional numerical solutions. The present investigation is conducted in a domain of normalized dimensions $L=10, W=5, h=1$ and $A=0.1$. The absorption of the solar radiation is assumed to be characterized by a single bulk attenuation coefficient $\eta=0.37$ (normalized by the maximum water depth). Only the initial and transitional stages of the three-stage flow development, which is normally expected in the convective flow regimes (Lei \& Patterson 2002a, b), are considered. 
For the above flow configurations, the critical Grashof number below which the thermal boundary layer is stable to the Rayleigh-Bénard instability is found to be between $5 \times 10^{3}$ and $10^{4}$, which agrees well with the earlier scaling result (Lei \& Patterson 2002b). The critical time at the onset of the Rayleigh-Bénard instability revealed from the direct stability analysis is also in an excellent agreement with the scaling prediction $\left(t_{B} \sim\left(R a_{c} / \operatorname{Pr} G r\right)^{1 / 2}\right)$.

For Grashof numbers in the convective regime (above the critical value), it is demonstrated that the critical time for the onset of the convective instability increases, whereas the growth rate of the perturbation as well as the dominant transverse wavenumber decrease, with the decrease of the Grashof number. As the Grashof number approaches the critical value, a zero growth rate is expected.

By means of single-mode perturbation, the preferred mode of the thermal boundary layer instability is determined for the case of $\mathrm{Pr}=6.83$ and $\mathrm{Gr}=2.51 \times 10^{6}$, corresponding to the parameters of a previous model experiment (Lei \& Patterson $2002 a$ ). It is found that the most unstable mode of the convective instability has a transverse wavenumber around 14 to 15 , and the most amplified mode has a transverse wavenumber around 12 to 13. It is also identified that the most unstable region is around $x=3.0$, independent of the perturbation mode. Similar procedures may be applied to any other flow conditions in order to determine the preferred mode of instability.

It is worth noting that in the present study the Grashof number is defined in terms of the maximum water depth. This is appropriate for the shallow water considered, for which the maximum water depth is of the order of, or less than, the length scale imposed by the absorption of incident radiation, i.e. the attenuation length. In this case, the heating by the bottom heat flux discussed earlier is dominant (Lei \& Patterson, 2002b). In geophysical situations such as a reservoir sidearm, the maximum water depth may be larger than the attenuation length, the Grashof number is much larger than those considered here, and the shallow water problem must be coupled with the stratification and mixing processes in deep waters. The dynamic processes in such situations are rather complicated. Large-scale models relevant to reservoirs are described in Fischer et al. (1979).

This research was financially supported by the Australian Research Council and James Cook University. The valuable comments from the anonymous reviewers on an earlier version of the manuscript are greatly appreciated.

\section{REFERENCES}

ARmfield, S. \& JANSSEN, R. 1996 A direct boundary-layer stability analysis of steady-state cavity convection flow. Intl J. Heat Fluid Flow 17, 539-546.

Asan, H. \& NAmLI, L. 2000 Laminar natural convection in a pitched roof of triangular cross-section: summer day boundary conditions. Energy and Buildings 33, 69-73.

Farrow, D. E. \& Patterson, J. C. $1993 a$ On the response of a reservoir sidearm to diurnal heating and cooling. J. Fluid Mech. 246, 143-161.

Farrow, D. E. \& Patterson, J. C. $1993 b$ On the stability of the near shore waters of a lake when subject to solar heating. Intl J. Heat Mass Transfer 36, 89-100.

Farrow, D. E. \& PAtTerson, J. C. 1994 The daytime circulation and temperature structure in a reservoir sidearm. Intl J. Heat Mass Transfer 37, 1957-1968.

Fischer, H. B., List, E. J., Koh, R. C. Y., Imberger, J. \& Brooks, N. H. 1979 Mixing in Inland and Coastal Waters, Academic.

Foster, T. D. 1971 Intermittent convection. Geophys. Fluid Dyn. 2, 201-217. 
Horsch, G. M. \& Stefan, H. G. 1988 Convective circulation in littoral water due to surface cooling. Limnol. Oceanogr. 33, 1068-1083.

Horsch, G. M., Stefan, H. G. \& Gavali, S. 1994 Numerical simulation of cooling-induced convective currents on a littoral slope. Intl J. Numer. Meth. Fluids 19, 105-134.

Hyun, J. M. 1994 Unsteady buoyant convection in an enclosure. Adv. Heat Transfer 24, 277-320.

JANSSEN, R. \& ARMFIELD, S. 1996 Stability properties of the vertical boundary layers in differentially heated cavities. Intl J. Heat Fluid Flow 17, 547-556.

KIRK, J. T. O. 1986 Optical limnology - a manifesto. In Limnology in Australia (ed. P. De Deckker \& W. D. Williams), pp. 33-62. CSIRO Australia and Dr W. Junk.

KURZWEG, U. H. 1970 Stability of natural convection within an inclined channel. Trans. ASME: J. Heat Transfer 92, 190-191.

Lei, C., Cheng, L. \& Kavanagh, K. 2001 Spanwise length effects on three-dimensional modelling of flow over a circular cylinder. Comput. Meth. Appl. Mech. Engng 190, 2909-2923.

Lei, C. \& Patterson, J. C. 2001 Two- and three-dimensional temperature structures in a shallow wedge subject to solar radiation. Proc. 14th Australasian Fluid Mechanics Conf., Adelaide, Australia, December 10-14, 2001, vol. I, pp. 359-362.

Lei, C. \& Patterson, J. C. $2002 a$ Natural convection in a reservoir sidearm subject to solar radiation: experimental observations. Exps. Fluids 32, 590-599.

Lei, C. \& Patterson, J. C. $2002 b$ Unsteady natural convection in a triangular enclosure induced by absorption of radiation. J. Fluid Mech. 460, 181-209.

Monismith, S. G., Imberger, J. \& Morison, M. L. 1990 Convective motions in the sidearm of a small reservoir. Limnol. \& Oceanogr. 35, 1676-1702.

Ostrach, S. 1988 Natural convection in enclosures. Trans. ASME: J. Heat Transfer 110, 1175-1190.

Poulikakos, D. \& Bejan, A. $1983 a$ The fluid dynamics of an attic space. J. Fluid Mech. 131, 251-269.

Poulikakos, D. \& Bejan, A. $1983 b$ Natural convection experiments in a triangular enclosure. Trans. ASME: J. Heat Transfer 105, 652-655.

RAbl, A. \& Nielsen, C. E. 1975 Solar ponds for space heating. Solar Energy 17, 1-12.

SALmun, H. 1995a Convection patterns in a triangular domain. Intl J. Heat Mass Transfer 18, $351-362$.

SALmun, H. $1995 b$ The stability of a single-cell steady-state solution in a triangular enclosure. Intl J. Heat Mass Transfer 18, 363-369. 